\title{
Reversible Jump MCMC Algorithm for Transformed Laplacian AR: Application in Modeling $\mathrm{CO}_{2}$ Emission Data
}

\author{
Suparman $^{1, *}$, Hery Suharna ${ }^{2}$, Mahyudin Ritonga ${ }^{3}$, Fitriana Ibrahim ${ }^{2}$, Tedy Machmud ${ }^{4}$, \\ Mohd Saifullah Rusiman ${ }^{5}$, Yahya Hairun ${ }^{2}$, Idrus Alhaddad ${ }^{2}$ \\ ${ }^{1}$ Faculty of Teacher Training and Education, Universitas Ahmad Dahlan, Indonesia \\ ${ }^{2}$ Faculty of Teacher Training and Education, Universitas Khairun, Ternate, Indonesia \\ ${ }^{3}$ Faculty of Islamic Studies, Muhammadiyah University of West Sumatera, Padang, Indonesia \\ ${ }^{4}$ Faculty of Mathematics and Sciences, Universitas Negeri Gorontalo, Gorontalo, Indonesia \\ ${ }^{5}$ Faculty of Applied Sciences and Technology, Universiti Tun Hussein Onn Malaysia, Malaysia
}

Received November 26, 2021; Revised January 21, 2022; Accepted February 16, 2022

\section{Cite This Paper in the following Citation Styles}

(a): [1] Suparman, Hery Suharna, Mahyudin Ritonga, Fitriana Ibrahim, Tedy Machmud, Mohd Saifullah Rusiman, Yahya Hairun, Idrus Alhaddad, "Reversible Jump MCMC Algorithm for Transformed Laplacian AR: Application in Modeling $\mathrm{CO}_{2}$ Emission Data," Mathematics and Statistics, Vol. 10, No. 2, pp. 301 - 307, 2022. DOI: $10.13189 / \mathrm{ms} .2022 .100203$.

(b): Suparman, Hery Suharna, Mahyudin Ritonga, Fitriana Ibrahim, Tedy Machmud, Mohd Saifullah Rusiman, Yahya Hairun, Idrus Alhaddad, (2022). Reversible Jump MCMC Algorithm for Transformed Laplacian AR: Application in Modeling $\mathrm{CO}_{2}$ Emission Data. Mathematics and Statistics, 10(2), 301 - 307. DOI: 10.13189/ms.2022.100203.

Copyright $\bigcirc 2022$ by authors, all rights reserved. Authors agree that this article remains permanently open access under the terms of the Creative Commons Attribution License 4.0 International License

\begin{abstract}
Autoregressive (AR) model is applied to model various types of data. For confidential data, data confusion is very important to protect the data from being known by other unauthorized parties. This paper aims to find data modeling with transformations in the AR model. In this AR model, the noise has a Laplace distribution. AR model parameters include order, coefficients, and variance of the noise. The estimation of the AR model parameter is proposed in a Bayesian method by using the reversible jump Markov Chain Monte Carlo (MCMC) algorithm. This paper shows that the posterior distribution of AR model parameters has a complicated equation, so the Bayes estimator cannot be determined analytically. Bayes estimators for AR model parameters are calculated using the reversible jump MCMC algorithm. This algorithm was validated through a simulation study. This algorithm can accurately estimate the parameters of the transformed AR model with Laplacian noise. This algorithm produces an AR model that satisfies the stationary conditions. The novelty in this paper is the use of transformations in the Laplacian AR model to secure research data when the research results are published in a scientific journal. As an example application, the Laplacian AR model was used to
\end{abstract}

model $\mathrm{CO}_{2}$ emission data. The results of this paper can be applied to modeling and forecasting confidential data in various sectors.

Keywords Autoregressive Processes, Bayes Methods, $\mathrm{CO}_{2}$ Emission, Laplacian Noise, Monte Carlo Methods

\section{Introduction}

Autoregressive (AR) is a stochastic mathematical model used for forecasting and pattern recognition. According to [1] [2], the understanding and characteristics of this model are widely used in various fields, such as time series and signal processing. Also, it contains noise. This noise is assumed to have a certain distribution either in the form of multiplicative or additive. For example, the noise present in the AR model is in the form of additives, while in some literature such as [3], it is assumed to have a Gaussian or normal distribution. Moreover, for wider usage, models have been developed to be in addition to a normal distribution, such as Pareto noise [4], model AR 
exponential noise [5], and Laplace noise [6]. However, these models do not protect confidential data when the research results are published in a scientific journal, hence an additional method known as variable transformation is needed to secure the data.

Variable transformation is a method of changing a random variable into a new one and it is often used in parametric data analysis when the assumptions are not met [7]. For example, the initial data, $x$ is transformed into y through the transformation $y=h(x)$, meanwhile, this data $\mathrm{y}$ is used to secure $\mathrm{x}$. Furthermore, the modeling for data $\mathrm{x}$ is sought through the inverse transformation $x=h^{-1}(y)$, where transformation $\mathrm{h}$ is the key to securing the $\mathrm{x}$ data.

Therefore, variable transformation is used in the AR model to protect the confidentiality of the modeled data when the research results are published a scientific journal. This paper focuses on the Laplacian noise AR model to determine: (a) a transformation for the AR model with Laplacian noise, (b) the estimation for the order of the AR model, (c) the estimation for the coefficients, and (d) the estimation for the variance noise of the AR model.

\section{Literatur Study}

\subsection{Laplace Distribution}

The random variable $\mathrm{z}$ has a Laplace distribution with parameters $\mu$ and $\beta$ if the probability density function is as follows [8]:

$$
f(z \mid \mu, \beta)=\frac{1}{2 \beta} \exp -\frac{|z-\mu|}{\beta} .
$$

This Laplace distribution is also found in various kinds of literature including [9] and [10].

\subsection{AR Model with Laplace Noise}

In this paper, $x_{1}, \ldots, x_{n}$ represent a data set, where $n$ is the number of observations. The data is said to have an AR model of order $p$, when it meets the following stochastic equation [1]:

$$
\mathrm{x}_{\mathrm{t}}+\sum_{i=1}^{p} \phi_{i} x_{t-i}=\mathrm{z}_{\mathrm{t}}
$$

Here, $p$ represents the order of the AR model, and $\phi_{1}, \phi_{2}, \cdots, \phi_{p}$ represent the coefficients of the AR model. The variable random of $z_{1}, \ldots, z_{n}$ has a Laplace distribution with parameter $\mu$ where $\beta=0$. The use of this Laplace noise is found in various literatures, including [6] and [11].

\subsection{Variable Transformation}

Variable transformation is a method used to change a random variable into another one. For instance, $x$ is a continuous random variable, and $f(x)$ is a probability density function for the random variable $x$. If $y=h(x)$ is an increasing or decreasing function, then the random variable $y=h(x)$ has a probability density function of [12]:

$$
g(y)=f\left[h^{-1}(y)\right]\left|\frac{d h^{-1}(y)}{d y}\right|
$$

\subsection{Reversible Jump MCMC}

In this section, $E$ and $\pi$ represent the set and probability of states, hence the reversible jump MCMC algorithm [13] is applied to produce a Markov chain at $E$ which has a stationary probability equal to $\pi$. In addition, $q$ declares an auxiliary kernel on $E$, and starting from $w \in E$, the drawing of a new point $w^{*}$ is carried out in two stages. First, point $w^{*}$ is drawn according to $q\left(w, w^{*}\right)$ while in the second stage, point $w^{*}$ is accepted with probability:

$$
\rho\left(w, w^{*}\right)=\min \left\{1, \frac{\pi\left(w^{*}\right) q\left(w^{*}, w\right)}{\pi(w) q\left(w, w^{*}\right)}\right\} .
$$

Therefore, in the reversible jump MCMC, it is possible to move the Markov chain between spaces where the spaces have different dimensions.

\section{Materials and Methods}

Data in this paper is time series data. This time series data is modeled by an AR model with Laplace noise. The following are the steps of data modeling. Let $x_{t}$ ( $t=$ $1,2, \cdots, n)$ be time series data. The data is transformed by using $y_{t}=a x_{t}$, The time series data $x_{t}$ is modeled as an AR model with Laplace noise. The likelihood function of times series data is determined. The prior distribution of the parameters is selected. The posterior distribution of the parameters is determined. Bayes estimators for parameters are calculated using the reversible jump MCMC algorithm.

\section{Results and Discussion}

\subsection{Likelihood Function}

Let $x=\left(x_{1}, \ldots, x_{n}\right)$ be the original data, it is modeled by the AR model. In this AR model, the noise has a Laplace distribution with parameter $\mu$ where $\beta=0$. Because the noise $z_{t}(t=1, \ldots, n)$ is assumed to have a Laplace distribution with parameter $\mu$ where $\beta=0$, the probability function for $x_{t}$ is written as:

$$
f\left(x_{t} \mid \beta\right)=\frac{1}{2 \beta} \exp -\frac{\left|\Sigma_{i=1}^{p} \phi_{i} x_{t-i}+x_{t}\right|}{\beta} .
$$

To make it confidential, the original data is firstly transformed using $\mathrm{y}=\mathrm{ax}$. If the time series $x_{t}$ is transformed to the time series $y_{t}=a x_{t}$, the probability function for $y_{t}$ is: 


$$
f\left(y_{t} \mid \beta\right)=\frac{1}{2 \mathrm{a} \beta} \exp -\frac{\left|\sum_{i=1}^{p} \phi_{i} y_{t-i}+y_{t}\right|}{\mathrm{a} \beta} .
$$

If $y=\left(y_{1}, \ldots, y_{n}\right)$ is the time series of the transformation results, the probability function for $y$ is [6]:

$$
\begin{aligned}
& f\left(y \mid p, \phi^{(p)}, \beta\right)=\left(\frac{1}{2 \mathrm{a} \beta}\right)^{n-p} \exp \\
& \quad-\frac{1}{a \beta} \sum_{t=p+1}^{n}\left|\sum_{i=1}^{p} \phi_{i} y_{t-i}+y_{t}\right|
\end{aligned}
$$

where $\phi^{(p)}=\left(\phi_{1}, \ldots, \phi_{p}\right)$.

According to [2], an AR model of order $p$ that satisfies the stationarity condition is important in time series. However, the coefficients were reparameterized because the stationarity condition of the AR was difficult to determine when the order of $p$ was large. For example, $r_{1}, r_{2}, \cdots, r_{p}$ represent partial autocorrelation functions that correspond to an AR model of order $p$. Also, $F$ represents a transformation from $\left(\phi_{1}, \ldots, \phi_{\mathrm{p}}\right) \in S_{p}$ to $\left(r_{1}, r_{2}, \cdots, r_{p}\right) \in(-1,1)^{p}$, where $S_{p}$ is the stationarity region of the AR model [14]. Through reparameterization, the probability function for the time series $y$ in $r_{1}, r_{2}, \cdots, r_{p}$ becomes [6]:

$$
\begin{aligned}
& f\left(y \mid p, r^{(p)}, \beta\right)=\left(\frac{1}{2 \mathrm{a} \beta}\right)^{n-p} \exp \\
& \quad-\frac{1}{a \beta} \sum_{t=p+1}^{n}\left|\sum_{i=1}^{p} F^{-1}\left(r_{i}\right) y_{t-i}+y_{t}\right|
\end{aligned}
$$

where $F^{-1}$ is the inverse transformation of $F$.

\subsection{Prior and Posterior Distributions}

The prior distribution for parameters is selected as in [6]. The prior distribution for the AR model order is a Binomial distribution with parameters $p_{\max }$ and $\lambda$. The prior distribution for the coefficients of the AR model is a uniform distribution at interval $(-1,1)^{p}$. While the prior distribution for the parameter $\beta$ is an inverse Gamma with parameter $v$ where $\mathrm{u}=2$. These prior distributions contain hyperparameters, namely $\lambda$ and $v$. The prior distribution for the hyperparameter $\lambda$ is a uniform distribution at the interval $(0,1)$. While the prior distribution for the hyperparameter $v$ is a Jeffreys prior distribution. Therefore, the joint distribution between the prior distribution of the parameters and the prior distribution of the hyperparameter can be written as [6]:

$$
\begin{aligned}
\pi\left(p, r^{(p)}, \lambda, \beta, v\right) & =C_{p}^{p_{\max }} \lambda^{p}(1-\lambda)^{p_{\max }-p} \frac{1}{2^{p}} \frac{v^{2}}{\beta^{3}} \exp \\
- & \frac{v}{\beta} \frac{1}{v} .
\end{aligned}
$$

The posterior distribution for $\left(p, r^{(p)}, \lambda, \beta, v\right)$ is expressed as follows [6]:

$$
\begin{gathered}
\pi\left(p, r^{(p)}, \lambda, \beta, v \mid x\right)=\left(\frac{1}{2 a}\right)^{n-p}\left(\frac{1}{\beta}\right)^{n-p+3} \exp - \\
\frac{1}{a \beta} \sum_{t=p+1}^{n}\left|\sum_{i=1}^{p} F^{-1}\left(r_{i}\right) y_{t-i}+y_{t}\right| C_{p}^{p_{\max }} \lambda^{p}(1- \\
\lambda)^{p_{\max }-p} \frac{1}{2^{p}} v \exp -\frac{v}{\beta} .
\end{gathered}
$$

\subsection{Reversible Jump MCMC Algorithm}

Posterior distribution simulation is carried out in two stages, namely conditional distribution simulation for $(\lambda, \beta, v)$ when given $\left(p, r^{(p)}\right)$ and conditional distribution simulation for $\left(p, r^{(p)}\right)$ when given $(\lambda, \beta, v)$. The first distribution is easily recognized by the inverse gamma distribution, beta distribution, and gamma distribution. Because of this, the simulation of $(\lambda, \beta, v)$ when given $\left(p, r^{(p)}\right)$ is carried out as follows [6]:

$$
\begin{gathered}
\beta \sim I G\left(n-p+2, v+\frac{1}{a} \sum_{t=p+1}^{n} \mid \sum_{i=1}^{p} F^{-1}\left(r_{i}\right) y_{t-i}+\right. \\
\left.y_{t} \mid\right), \lambda \sim B\left(p+1, p_{\text {max }}-p+1\right), v \sim G\left(2, \frac{1}{\beta}\right) .
\end{gathered}
$$

But, the conditional distribution for $\left(p, r^{(p)}\right)$ when given $(\lambda, \beta, v)$ is not recognized easily because parameter $\left(p, r^{(p)}\right)$ has a complex shape with a variable dimension space. Consequently, the simulation is carried out by using the reversible jump MCMC algorithm with three kinds of transformations, including coefficient change, order birth, and order death [6].

\subsection{Model Validation}

The model generated by the reversible jump MCMC algorithm was validated via a simulation study. First, the parameter values are set, then the simulation data was created based on the values of this model. Furthermore, the algorithm was implemented on the simulation data to estimate the values. Consequently, the model estimators were compared with the predefined values and the result is said to be true when the estimator is close to the value of the parameters that have been set previously.

For example, a time series of 250 is created based on equation (1) with the parameters of the AR model and the maximum order $p_{\max }=10$ as presented in Table 1 .

Table 1. Synthesis time-series parameters (before transformation)

\begin{tabular}{ccc}
\hline$p$ & $\left(\phi_{1}, \phi_{2}, \phi_{3}\right)$ & $\beta$ \\
\hline 4 & $(-0.79,-0.03,0.90,0.68)$ & 2 \\
\hline
\end{tabular}

Table 1 shows that the order of the model is 4 and the coefficients of the model are $(-0.79,-0.03,0.90,0.68)$. The resulting time series is presented in Figure 1. 


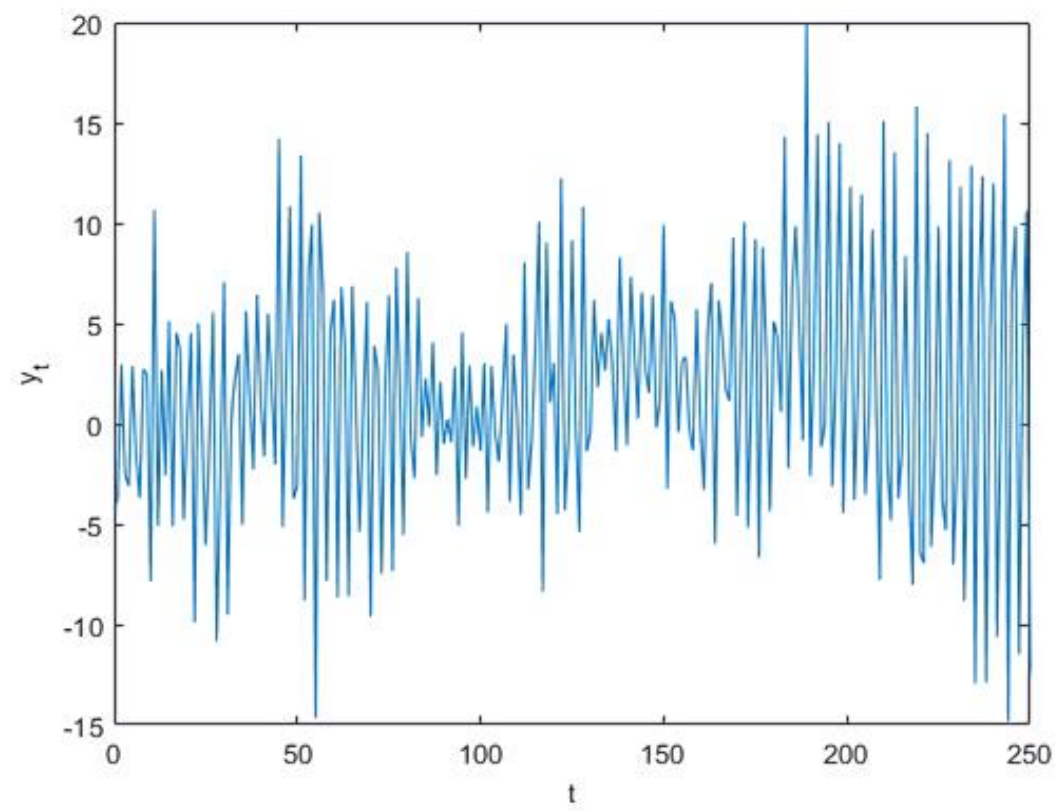

Figure 1. Synthesis data (before transformation)

Then, this data is transformed through $y=5 x$, and the model parameters in Table 1 change to that of Table 2 .

Table 2. Synthesis time-series parameters (after transformation)

\begin{tabular}{ccc}
\hline$p$ & $\left(\phi_{1}, \phi_{2}, \phi_{3}\right)$ & $\beta$ \\
\hline 4 & $(-0.79,-0.03,0.90,0.68)$ & 10 \\
\hline
\end{tabular}

The resulting time series is presented in Figure 2.

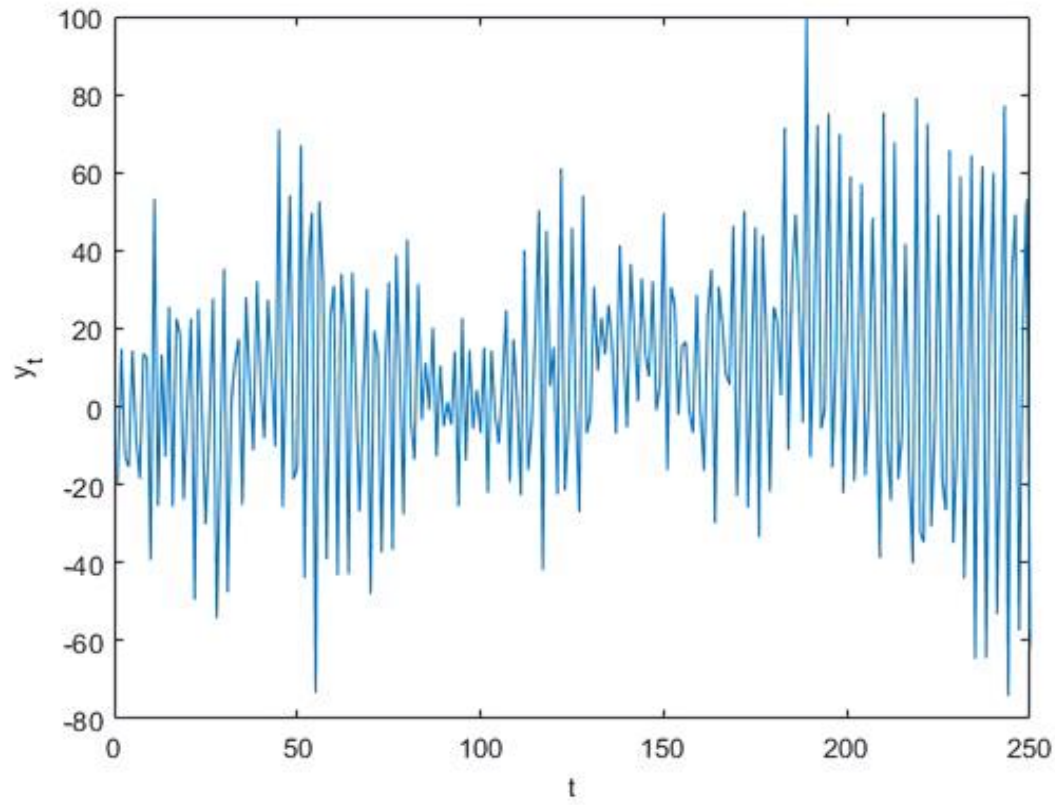

Figure 2. Synthesis data (after transformation) 


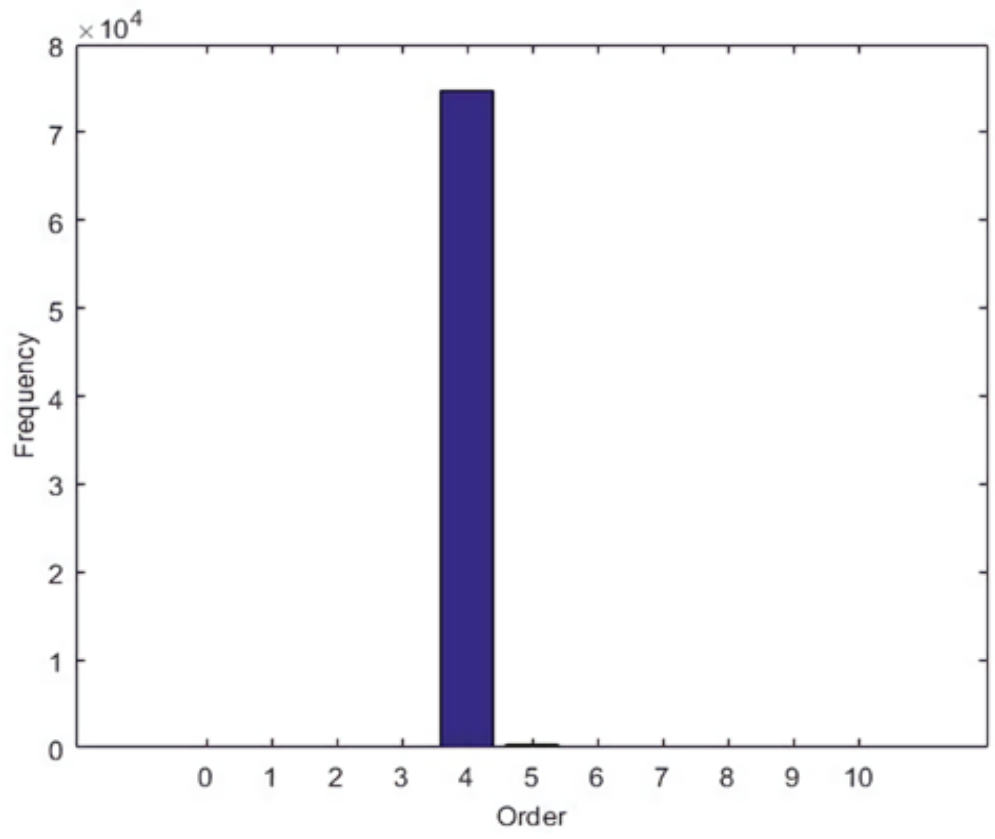

Figure 3. Histogram for the order $p$

Furthermore, the synthesis data (after transformation) was used as input for the reversible jump MCMC algorithm and was run for 100,000 iterations with a burn-in period of 25,000. The output was an AR model parameter estimator and its order is presented in Figure 3.

The estimation results of model parameters are also presented in Table 3.

Table 3. Estimation of synthesis time-series parameters (after transformation)

\begin{tabular}{ccc}
\hline$\hat{p}$ & $\left(\hat{\phi}_{1}, \hat{\phi}_{2}, \hat{\phi}_{3}, \hat{\phi}_{4}\right)$ & $\hat{\beta}$ \\
\hline 4 & $(-0.78,-0.02,0.92,0.70)$ & 12.50 \\
\hline
\end{tabular}

\subsection{Applications in Modeling Carbon Dioxide $\left(\mathrm{CO}_{2}\right)$ Emission Data}

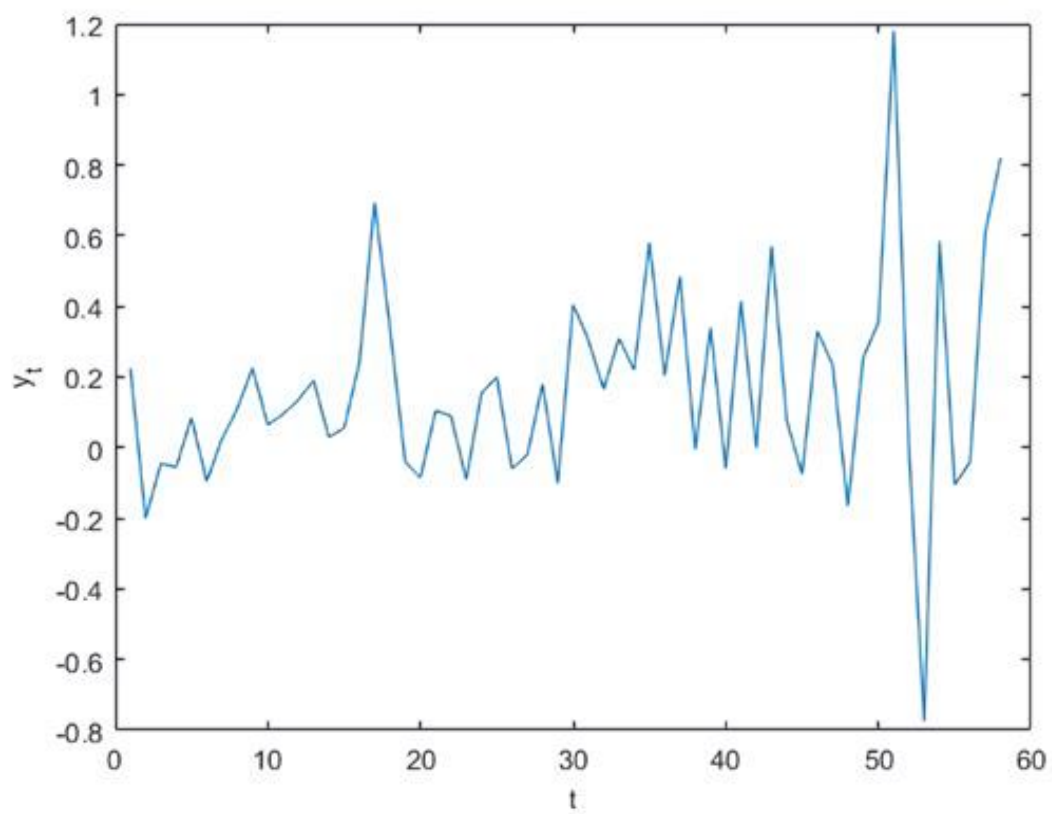

Figure 4. $\mathrm{CO}_{2}$ emission data of a country 


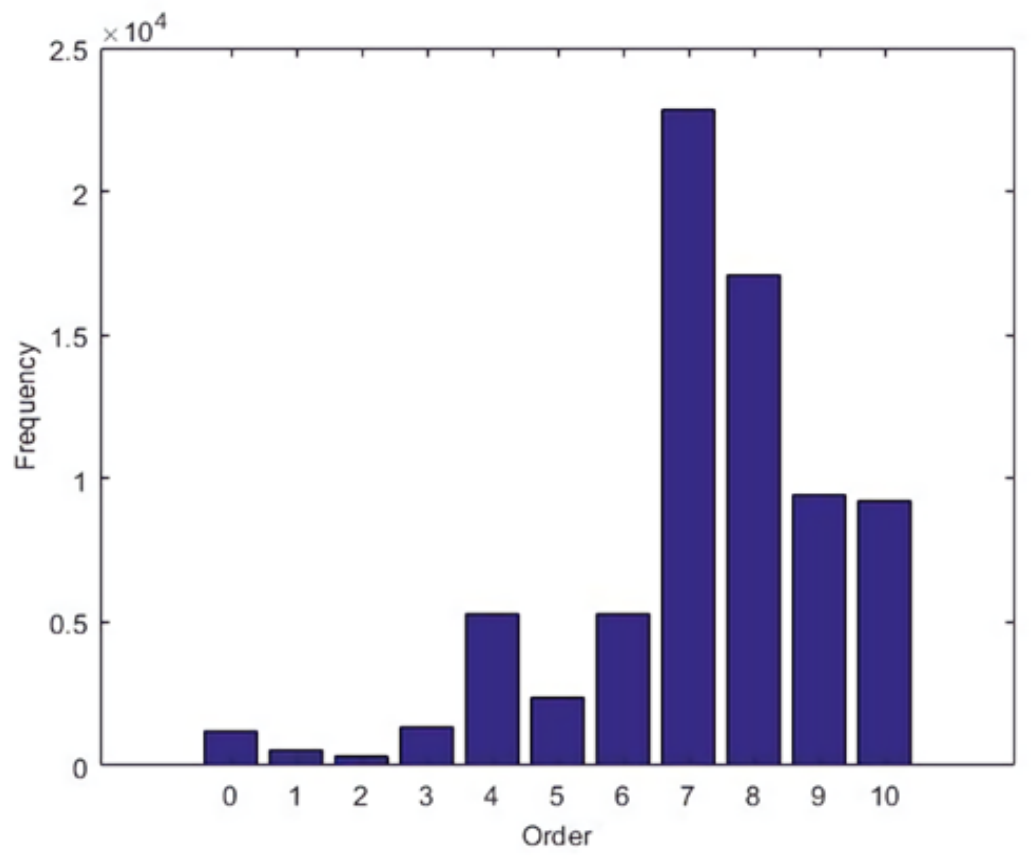

Figure 5. Histogram for the order $p$

The Laplacian AR model used to model $\mathrm{CO}_{2}$ emission data (metric tons per capita) was taken from https://data.worldbank.org/indicator/EN.ATM.CO2E.PC in a country between 1960 to 2018. The differentiation of $\mathrm{CO}_{2}$ emission was carried out to obtain stationary data before being transformed into new $\mathrm{CO}_{2}$ as presented in Figure 4.

Furthermore, the $\mathrm{CO}_{2}$ emission data was used as input to the reversible jump MCMC algorithm and was run for 100,000 iterations with a burn-in period of 25,000 iterations. The output was an estimator of the parameters of the Laplacian AR model as shown in Figure 5.

Meanwhile, Table 4 shows the estimation of model parameters.

Table 4. Parameter estimation for $\mathrm{CO}_{2}$ emission data

\begin{tabular}{ccc}
\hline$\hat{p}$ & $\left(\hat{\phi}_{1}, \hat{\phi}_{2}, \hat{\phi}_{3}, \hat{\phi}_{4}, \hat{\phi}_{5}, \hat{\phi}_{6}, \hat{\phi}_{7}\right)$ & $\hat{\beta}$ \\
\hline 7 & $(-0.02,0.09,0.09,0.24,-0.23,0.30,0.34)$ & 0.26 \\
\hline
\end{tabular}

\subsection{Discussion}

Figure 3 shows that the highest order is reached at the value of $p=4$. This means that the estimator of the order of $\mathrm{p}$ is $\hat{p}=4$. Furthermore, the model coefficient estimators corresponding to $\hat{p}=4$ are presented in Table 3. If Table 2 and Table 3 are compared, the estimated parameter values in Table 3 are close to the true parameter values in Table 2 . These results indicate that the reversible jump MCMC algorithm can accurately estimate the parameters of the Laplacian AR model.

Figure 4 shows that the highest order is reached at the value of $p=7$. This means that the estimator of the order of $\mathrm{p}$ is $\hat{p}=7$. Furthermore, the model coefficient estimators corresponding to $\hat{p}=7$ are presented in Table 4. These results indicate that the $\mathrm{CO}_{2}$ emission data can be modeled by an AR model of order 7 with coefficient values as in Table 4 (second row, second column).

If the results of the research in this paper are compared with the results of research in [3], the results of this paper have similarities and differences. The similarity between the results of this paper and the results of research in [3] are both using the same prior distribution and the parameter estimation method using the reversible jump MCMC algorithm. The difference between the results of this paper and the results of research in Suparman is that this paper uses Laplace noise, while research in [3] uses Gaussian noise.

If the results of the research in this paper are compared with the results of research in [6] and [11] then this paper also has similarities and differences. The similarities between the results of this paper and the results of research in [6] and [11] are the three studies using the same prior distribution, the same noise distribution (Laplacian noise), reversible jump MCMC algorithm. While the difference between the results of this paper and the results of research in [6] is that this paper uses data transformed through the transformation $\mathrm{y}=\mathrm{ax}$ and the results of the paper are applied to $\mathrm{CO}_{2}$ data, but research in [6] uses data that is not transformed and the results of the paper were applied to the heart rate data. On the other hand, the difference between this paper and internal research [11] is that this paper uses data transformed through the $\mathrm{y}=$ ax transformation, the estimation method uses the reversible jump MCMC algorithm and the results of the paper are applied to $\mathrm{CO}_{2}$ data, but research in [11] using data that is not transformed, 
the estimation method uses the bootstrap reversible jump MCMC algorithm, and the results of the paper are applied to stock data.

In this paper, the transformation used is the transformation $y=a x$. To further maintain the confidentiality of the original data, this paper was developed using a more complicated transformation. Research results can be applied in a wider field because research results can not only be used in fields where data is not confidential but can also be applied to fields where data is confidential.

\section{Conclusion}

This paper examines the estimation procedure of the Laplacian AR model with the target data used being kept confidential by using the transformation method. The focus was on the linear transformation that passes through the coordinate center. However, the non-linear transformation has not been investigated, thus further research needs to be carried out to maintain more confidential data. This finding had been able to maintain data confidentiality and the order of the AR model, hence it is more accurate than when the value is determined subjectively. Consequently, the results need to be applied to a wider field because they are useful for modeling both confidential and non-confidential data. It is also recommended to use data modeling that protects data to ensure its confidentiality.

\section{Acknowledgments}

The authors are grateful to the reviewers for their comments and suggestions in improving this paper.

\section{REFERENCES}

[1] G. E. P. Box, G. M. Jenkins, G. C. Reinsel, and G. M. Ljung, Time Series Analysis: Forecasting and Control, 5th ed. John Wiley \& Sons, 2015.

[2] P. J. Brockwell and R. A. Davis, Times Series: Theory and Methods. New York: Springer, 1991.
[3] Suparman and M. S. Rusiman, "Hierarchical Bayesian Estimation for Stationary Autoregressive Models Using Reversible Jump MCMC Algorithm," Int. J. Eng. Technol., vol. 7, pp. 64-67, 2018, [Online]. Available: www.sciencepubco.com/index.php/IJET.

[4] J. Jureckova, H. L. Koul, and J. Picek, "Testing the tail index in autoregressive models," Ann Inst Stat Math, vol. 61, pp. 579-598, 2009.

[5] L. Larbi and H. Fellag, "Robust Bayesian Analysis of an Autoregressive Model with Exponential Innovations," Afr. Stat., vol. 11, no. 1, pp. 955-964, 2016.

[6] Suparman and A. M. Diponegoro, "Mathematical Model with Laplace Autoregressive Process for Heart Rate Signals," Technol. Reports Kansai Univ., vol. 62, no. 03, pp. 1001-1011, 2020.

[7] D. K. Lee, "Data transformation: A focus on the interpretation," Korean J. Anesthesiol., vol. 73, no. 6, pp. 503-508, 2020, doi: 10.4097/kja.20137.

[8] H. I. Okagbue, P. E. Oguntunde, A. A. Opanuga, and P. I. Adamu, "Laplace distribution: Ordinary differential equations," in Proceedings of the World Congress on Engineering and Computer Science, 2018, vol. 1, pp. 1-7.

[9] A. Minchole, L. Sornmo, and P. Laguna, "Detection of body position changes from the ECG using a Laplacian noise model," Biomed. Signal Process. Control, vol. 14, pp. 189-196, 2014.

[10] F. I. Miertoiu and B. Dumitrescu, "Feasibility pump algorithm for sparse representation under Laplacian noise," Math. Probl. Eng., vol. 2019, 2019, doi: $10.1155 / 2019 / 5615243$.

[11] Suparman, Y. Hairun, I. Alhaddad, T. Machmud, H. Suharna, and M. S. Rusiman, "Forecasting Software Using Laplacian AR Model based on Bootstrap-Reversible Jump MCMC : Application on Stock Price Data," Webology, vol. 18, no. Special Issue, pp. 1045-1055, 2021, doi: 10.14704/WEB/V18SI04/WEB18180.

[12] D. D. Wackerly, I. Mendenhall, and R. L. Scheaffer, Mathematical Statistics with Applications. Thomson Higher Education, 2008.

[13] P. J. Green, "Reversible jump Markov chain Monte Carlo computation and Bayesian model determination," Biometrika, vol. 82, no. 4, pp. 711-743, 1995.

[14] O. Barndorff-Nielsen and G. Schou, "On the Parametrization of Autoregressive models by partial autoccorelation," J. Multivar. Anal., vol. 3, pp. 408-419, 1973 